\title{
El síndrome de apnea obstructiva del sueño como factor de riesgo para enfermedades cardiovasculares y su asociación con hipertensión pulmonar
}

\author{
Jaime Eduardo Morales-Blanhir, ${ }^{*} \bowtie$ Matilde Valencia-Flores, ${ }^{*}$ Oscar Arturo Lozano-Cruz ${ }^{*}$ \\ *Médico Neumólogo, Director Médico de la Federación Mexicana de Enfermedades Raras; 'Instituto Nacional de Ciencias Médicas y \\ Nutrición Salvador Zubirán, Ciudad de México. \\ Trabajo recibido: 26-V-2015; aceptado: 24-I-2017
}

\begin{abstract}
RESUMEN. Existe evidencia sólida de que el síndrome de apnea obstructiva del sueño (SAOS) es un factor de riesgo cardiovascular. Los eventos de obstrucción de la vía aérea durante el sueño predisponen a alteraciones como hipertensión arterial sistémica, cardiopatía isquémica y enfermedad vascular cerebral, representando éstas un impacto negativo en la salud pública de nuestro medio. EI SAOS se asocia, además, con disfunción endotelial, arritmias, insuficiencia cardíaca e hipertensión pulmonar (HP). Estas asociaciones han generado el desarrollo constante de evidencia que permite comprender el impacto independiente de este síndrome. En esta revisión de la literatura publicada en MEDLINE que es la base de datos más importante de la National Library of Medicine, tiene como objetivo mostrar al SAOS como un factor de riesgo cardiovascular, con especial atención a su asociación con el desarrollo de HP por ser un factor predisponente de disfunción ventricular.
\end{abstract}

Palabras clave: Enfermedad cardiovascular, hipertensión pulmonar, síndrome de apnea obstructiva del sueño.

ABSTRACT. Solid evidence shows that obstructive sleep apnea (OSA) is independently associated with cardiovascular risk. Airway obstruction during sleep predispose to cardiopulmonary disorders such as systemic hypertension, coronary artery disease and stroke, representing a negative impact on public health. Also, OSA has been associated with the development of endothelial dysfunction, arrhythmias, heart failure and pulmonary hypertension (PH). These associations have promoted the development of evidence that allows to understand the independent impact of this syndrome. This review of the literature published in MEDLINE, which is the most important database of the National Library of Medicine, aims to show the role that OSA has as cardiovascular risk and focus on its association with the development of pulmonary hypertension for being a predisposing factor for ventricular dysfunction.

Key words: Cardiovascular disease, obstructive sleep apnea syndrome, pulmonary arterial hypertension.

\section{INTRODUCCIÓN}

A través de estudios clínicos el síndrome de apnea obstructiva del sueño (SAOS) se ha asociado con diversas alteraciones cardiovasculares ${ }^{1-3}$ como la hipertensión arterial sistémica, ${ }^{4}$ cardiopatía isquémica, ${ }^{1}$ enfermedad vascular cerebral, ${ }^{5}$ trastornos del ritmo cardíaco, ${ }^{6}$ insuficiencia cardíaca ${ }^{7}$ e hipertensión pulmonar $(\mathrm{HP})^{8}$ (tabla 1).

La patogénesis de las enfermedades cardiovasculares comparten factores de riesgo con el SAOS. Lo anterior ha producido resultados controversiales y el desarrollo constante de estudios que en la actualidad apoyan de manera importante el papel del SAOS como un factor de riesgo independiente para el desarrollo de enfermedades cardiovasculares. ${ }^{9}$
EI SAOS se caracteriza por eventos de obstrucción de la vía aérea que conducen a episodios de hipoxemia, cambios de presión intratorácica y activación del sistema simpático. ${ }^{10}$ Estos mecanismos durante el sueño conducen a un aumento de la presión arterial sistémica, incremento de la poscarga ventricular y disminución del gasto cardíaco; ${ }^{11,12}$ y de forma crónica conducen a disfunción endotelial, arritmias cardíacas, hipertensión arterial sistémica diurna sostenida, HP e insuficiencia cardíaca. ${ }^{13}$

Las alteraciones en la respiración durante el sueño repercuten en la circulación pulmonar, se han reconocido a la hipoxia y la hipercapnia como los principales mecanismos; aunque también participan la disfunción ventricular, la generación de productos de oxidación, el aumento de niveles de endotelina 1 y la reducción 
Tabla 1. Prevalencia reportada de SAOS en enfermedades cardiovasculares y riesgo de morbilidades en pacientes con SAOS. , $^{1,329,38,44}$

$\left.\begin{array}{lcc}\hline \text { Morbilidad } & \text { Prevalencia de SAOS }(\%)^{3} & \text { Riesgo en pacientes con SAOS } \\ \hline \text { HAS } & 30-83 \% & \text { OR } 1.37 \\ \text { EVC } & 43-91 \% & (\text { IC } 95 \%=1.03-1.83)^{38} \\ \text { OR } 1.97\end{array}\right)$

SAOS = síndrome de apnea obstructiva del sueño; HAS = hipertensión arterial sistémica; EVC = evento vascular cerebral; OR = odds ratio; IC = intervalo de confianza.

Tomado y modificado de: Floras JS. 2014. ${ }^{3}$

del óxido nítrico alveolar. ${ }^{12}$ Lo anterior contribuye a la prevalencia alta de HP en pacientes con SAOS. ${ }^{14}$

\section{SÍNDROME DE APNEA OBSTRUCTIVA DEL SUEÑO}

\section{Definición y epidemiología del SAOS}

Hace medio siglo se desconocía el SAOS y por consecuencia su impacto. Sin embargo, durante las últimas décadas su relevancia es evidente debido a que representa una causa significativa de morbilidad y mortalidad..$^{15}$ La implementación de la polisomnografía en las clínicas del sueño permite identificar alteraciones que ocurren durante el sueño y establecer criterios diagnósticos para SAOS. La importancia de realizar el diagnóstico temprano permite administrar la terapia con presión positiva en la vía aérea en diferentes modalidades (CPAP, bi-nivel, auto-CPAP, servo-ventilación); y de esta manera modificar los mecanismos fisiopatológicos que alteran la arquitectura del sueño y la función cardiopulmonar (figuras 1 y 2).

Actualmente la Academia Americana de Medicina del Sueño (AAMS) define al SAOS como la presencia de cinco o más eventos respiratorios por hora de sueño (índice apnea/hipopnea [IAH]) con síntomas o comorbilidades, o como la presencia de 15 eventos respiratorios por hora de sueño independiente de los síntomas y comorbilidades. ${ }^{16}$

Los estudios poblacionales disponibles concluyen que la prevalencia del SAOS con IAH bajo (superior a 5 y menor a 15) en adultos de la población general es de $9 \%$ en mujeres (95\% IC 5.6 a 12.0 ) y $24 \%$ en hombres (IC $95 \%$ $=19-28$ ); y para un IAH mayor de 15 es $4 \%$ en mujeres (IC 95\% = 1.5-6.6) y 9\% en hombres (IC 95\% = 6.4-11.0). ${ }^{17}$

La prevalencia del SAOS resulta todavía mayor en los pacientes con comorbilidades como el síndrome metabólico y enfermedad cardiovascular. ${ }^{2,18}$ Su prevalencia es entre 30 a 90\% en hipertensión sistémica, ${ }^{4,19}$ hipertensión refractaria, ${ }^{20}$ fibrilación auricular ${ }^{21}$ y síndrome metabólico. ${ }^{22}$

Se considera que la alta prevalencia del SAOS en pacientes con enfermedad cardiovascular establecida se debe a que se comparten varios factores de riesgo como son: mayor edad, vida sedentaria, sexo masculino y en especial la obesidad. ${ }^{23}$

Desde 1960 se vinculó la obesidad con alteraciones del sueño a través del síndrome de Pickwick. Estudios recientes fundamentados en el aumento de la prevalencia de obesidad en el mundo occidental continúan apoyando esta relación ${ }^{24} \mathrm{y}$ favorecen que las medidas de prevención y manejo de la obesidad sean prioridad en estos pacientes.

En América Latina se han diseñado estudios que permiten conocer la prevalencia de manera aproximada. Torre-Bouscoulet et al. estimaron la frecuencia de síntomas relacionados con SAOS como el ronquido en el $60 \%$, somnolencia diurna en el $16 \%$ y $12 \%$ con apneas durante el sueño, concluyendo que existe una alta prevalencia de SAOS sintomático en la muestra estudiada en la Ciudad de México y Área Metropolitana. ${ }^{25}$ Además en la Ciudad de México estudiaron la presencia combinada de ronquido, apnea y somnolencia diurna, esto les permitió concluir que la prevalencia del SAOS es $2.2 \%$ en mujeres y $4.4 \%$ en hombres. ${ }^{25}$ De acuerdo con los datos publicados por el Instituto Nacional de Ciencias Médicas y Nutrición (INCMNSZ, Ciudad de México), en sujetos obesos la prevalencia de SAOS es mayor a $80 \% .^{26}$

Los trastornos del sueño en poblaciones latinas son comunes y debe considerarse que el comportamiento del SAOS es variable dependiendo de la altitud en la que reside la población estudiada, se ha demostrado que la aparición de apneas centrales junto con apneas obstructivas es común en altitudes por encima de 1,600 

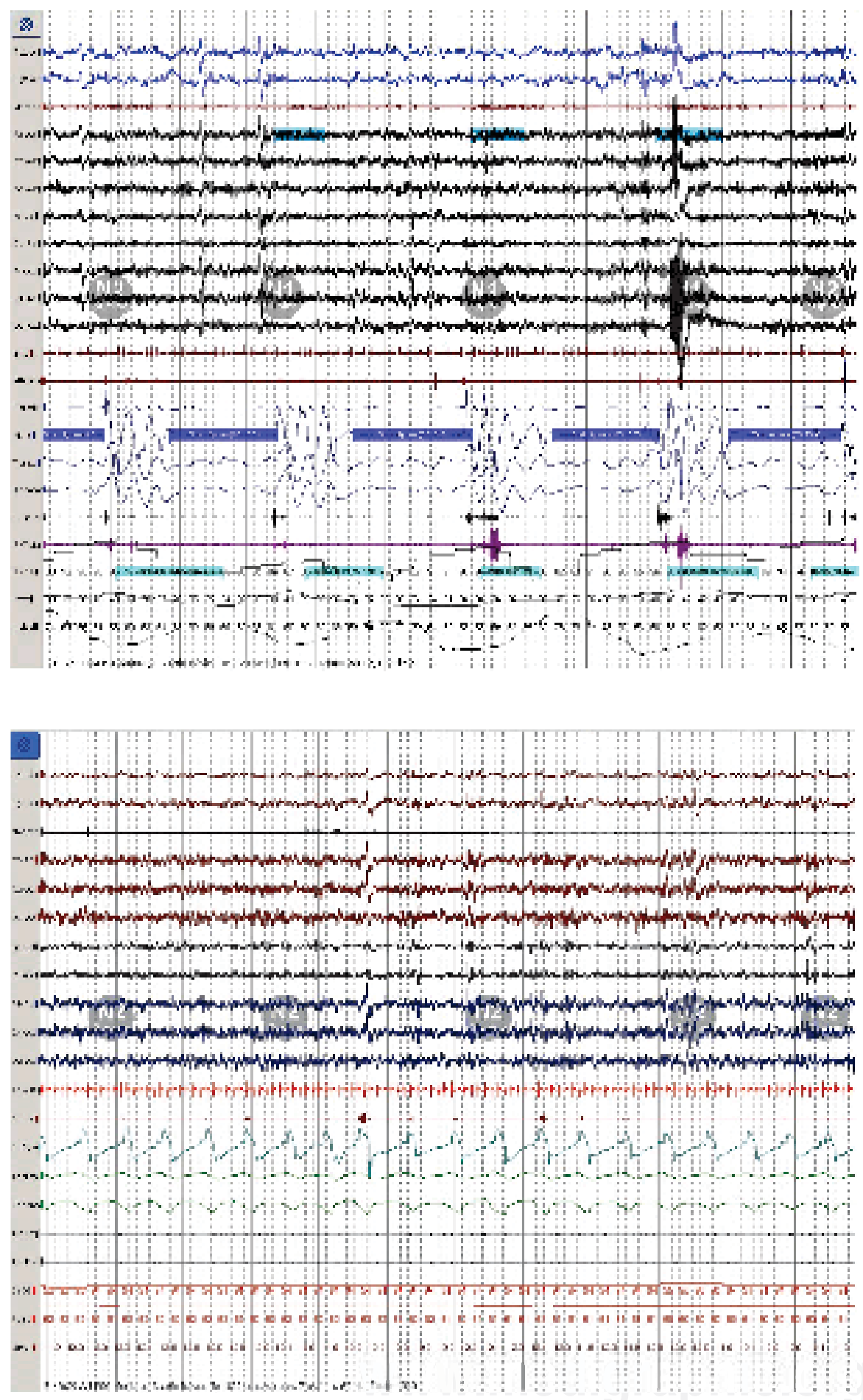

\section{Figura 1.}

Trazo de polisomnografía en etapa de sueño NMOR comprimido a $2 \mathrm{~min}$. Se observan períodos de apnea tipo obstructiva acompañadas de disminución en la saturación de oxígeno y variabilidad de frecuencia cardíaca. Consecutivamente ocurren microdespertares que restablecen la saturación de oxígeno.

\section{Figura 2.}

Trazo de polisomnografía en etapa de sueño NMOR durante el período terapéutico con CPAP. Se observa la desaparición de apneas obstructivas y variabilidad de la frecuencia cardíaca que permiten la consolidación del sueño.

CPAP = Presión positiva continua a la vía aérea. metros sobre el nivel del mar, ${ }^{27}$ como la Ciudad de México. En este sentido, el reconocimiento de apneas centrales implicaría otras estrategias en el tratamiento. No obstante, en la población de obesos en México el patrón que se ha observado es en su mayoría de eventos obstructivos, con pocas apneas centrales o mixtas. ${ }^{28}$
Es importante señalar que la baja prevalencia de apneas centrales en la cohorte estudiada en el INCMNSZ no es de sorprender, dado que los pacientes están habituados a vivir a una altitud moderada. Este hallazgo de una baja prevalencia de apneas centrales está en línea con otro reciente informe del grupo de Bogotá quienes reportan 
una prevalencia despreciable de apneas centrales en su cohorte de 988 pacientes referidos al Laboratorio de Sueño por sospechas de trastornos respiratorios del sueño. El índice de apnea central del sueño fue bajo, tanto para el grupo sin tratamiento como para el grupo con tratamiento con presión positiva y eventos centrales emergentes durante el tratamiento. ${ }^{29}$ Estos datos con los probables efectos de adaptación que ocurren en las poblaciones de residentes habituales en altitudes como las de la Ciudad de México y Bogotá, contrasta con la prevalencia de apneas centrales que puede ser mucho más alta en pacientes con y sin OSA (del inglés obstructive sleep apnea) que ascienden agudamente a alta altitud..$^{30}$

\section{IMPACTO DEL SAOS EN ENFERMEDADES CARDIOVASCULARES}

\section{Disfunción endotelial}

La disfunción endotelial se considera como un marcador temprano de daño vascular y el punto de partida para el desarrollo de enfermedades cardiovasculares. En el metaanálisis realizado por Wang et al. se evaluó el riesgo de enfermedad cardiovascular en pacientes con SAOS, se concluyó que esta población tiene un riesgo relativo de $1.79(\mathrm{IC} 95 \%=1.47-2.18)^{31}$ y un riesgo relativo de enfermedad isquémica coronaria de 3.1 (IC $95 \%=1.2-8.3){ }^{32}$

Los períodos de hipoxia y reoxigenación intermitente durante el sueño en los pacientes con SAOS generan un desequilibrio entre factores antioxidantes y oxidantes que se traduce en estrés oxidativo. Este estado favorece la formación de especies reactivas de oxígeno (ERO) que generan un estado proinflamatorio y conducen a una disfunción endotelial en estos pacientes. ${ }^{33}$

A su vez, las ERO producen la activación del factor nuclear Kappa B, que estimula la producción de mediadores inflamatorios como el factor de necrosis tumoral, IL6, IL8 y proteína C reactiva ${ }^{34}$ disminuyendo los niveles de óxido nítrico y, en consecuencia, la capacidad vasodilatadora. Lo anterior se corroboró mediante la medición de nitritos en el plasma de los pacientes con SAOS, donde se observaron niveles más bajos comparando con la población sin SAOS. ${ }^{35}$

Además, los pacientes se ven afectados al presentar mayor capacidad aterogénica, esto se demostró mediante el aumento del grosor carotídeo en la capa íntima y media, ${ }^{36}$ y por la mayor prevalencia de eventos cerebrovasculares silentes. ${ }^{37} \mathrm{El}$ tratamiento con CPAP ha evidenciado ser favorable en los pacientes con SAOS debido a que produce disminución en los niveles de varios mediadores proinflamatorios ${ }^{38}$ y adelgazamiento en el grosor de la íntima. ${ }^{39}$
Recién se han publicado los resultados de un estudio que compara el beneficio del CPAP para prevenir eventos cardiovasculares en pacientes con enfermedad cardiovascular establecida. El estudio mostró que el tratamiento con CPAP reduce los síntomas diurnos, mejora la calidad de vida y rendimiento laboral; pero no hay diferencia significativa en la prevención de eventos cardiovasculares recurrentes en pacientes con SAOS. ${ }^{40}$ Hay que señalar que la mayoría de los pacientes pertenecía a una etnia asiática y más de la mitad de ellos estaban bajo tratamiento con estatinas, las cuales se sabe tienen un efecto antioxidante que podría beneficiar a condiciones con estrés oxidativo como es el caso del SAOS. ${ }^{41}$ Con lo anterior, podemos pensar que en los casos más severos de la enfermedad se deben enfatizar las acciones para el diagnóstico temprano y realizar estudios en las formas tempranas de la enfermedad.

\section{Hipertensión arterial sistémica}

La hipertensión arterial sistémica (HAS) es frecuente en los pacientes con SAOS, con una prevalencia estimada entre 30 y $40 \%, 42$ lo anterior tiene relevancia clínica debido a que el SAOS representa hasta un rango del $64-84 \%$ de hipertensión arterial resistente. ${ }^{43}$

Se ha evaluado el riesgo independiente que tienen los pacientes con SAOS para desarrollar HAS. Mediante la comparación entre pacientes con y sin SAOS los resultados indican que esta población tiene mayor riesgo de desarrollar HAS, OR 1.37 (95\% 1.03-1.83). ${ }^{44}$ También se ha identificado que la severidad del SAOS incrementa el riesgo de desarrollar HAS como lo muestran Peppard et al.:4 IAH entre 0.1-4.9, OR = 1.42 (IC 95\% = 1.13-1.78); IAH entre 5-14.9, OR = $2.03($ IC $95 \%=1.29-3.17)$ y con IAH mayor o igual a $15, \mathrm{OR}=2.89(\mathrm{IC} 95 \%=1.46-5.64)$.

Se ha identificado que la actividad simpática aumentada en estos pacientes evita la disminución normal de la presión arterial durante el sueño. ${ }^{45}$ Además, se ha descrito la correlación entre el aumento en la concentración de aldosterona plasmática y la gravedad del síndrome que se relaciona también con hipertensión arterial resistente. ${ }^{46}$

El tratamiento con CPAP en los pacientes con SAOS ha mostrado resultados favorables para la presión arterial sistólica con diminución de 2 a $10 \mathrm{mmHg},{ }^{47}$ y como lo muestra el recién metaanálisis realizado por Fava et al. en el que se evidencia que la terapia con CPAP se asocia a reducción significativa de la tensión arterial sistólica y diastólica. ${ }^{48}$

\section{Enfermedad vascular cerebral}

La enfermedad vascular cerebral (EVC) representa un problema de salud pública debido a que es la segunda 
causa de muerte en el mundo y la principal causa de discapacidad a largo plazo. ${ }^{49} \mathrm{~A}$ pesar de compartir comorbilidades se ha informado que el riesgo independiente de EVC en paciente con SAOS es OR 1.97 (IC $95 \% 1.12-3.48)^{50}$ y este riesgo aumenta con la gravedad del SAOS hasta OR 4.33 (IC 95\% 1.32-14.24). ${ }^{51}$

Aunque el mecanismo exacto continúa en investigación, algunos estudios muestran que el inicio de terapia con presión positiva produce un efecto positivo sobre la supervivencia a largo plazo en pacientes con EVC isquémico $^{52}$ y disminuye la mortalidad en pacientes que toleran el CPAP (HR 1.58) en comparación con los que no lo toleran (HR 2.69). ${ }^{53}$ Pero hoy, la evidencia indica que la terapia con CPAP pudiera no ofrecer una diferencia significativa en prevenir la recurrencia de eventos cardiovasculares. ${ }^{40}$

\section{Insuficiencia cardíaca}

A pesar de los avances terapéuticos en insuficiencia cardíaca (IC), se reconoce que es un padecimiento con mortalidad superior al $50 \%$ a los cinco años después del diagnóstico. ${ }^{54}$ Por lo anterior, es importante identificar y tratar las condiciones que pueden contribuir a la aparición y progresión de esta enfermedad. ${ }^{55} \mathrm{En}$ esta revisión se sugiere que una de esas condiciones a investigar es el SAOS.

En ese sentido se reconoce que durante los períodos de apnea, la presión negativa intratorácica generada contra la faringe ocluida produce un aumento de la presión transmural del ventrículo derecho (VD) que ocasiona aumento en la poscarga. ${ }^{56}$ También se aumenta el retorno venoso y con esto la precarga del VD mientras que la vasoconstricción pulmonar, causada por la hipoxemia, aumenta la poscarga del VD. ${ }^{57}$ Además, la distención del VD y la desviación septal hacia la izquierda durante la diástole disminuye el llenado del ventrículo izquierdo. ${ }^{58}$

La combinación de los eventos mencionados anteriormente produce reducción del volumen sistólico y del gasto cardíaco, ${ }^{59}$ esto sin tratamiento, puede contribuir al desarrollo o progresión de la remodelación, hipertrofia e IC. Se ha estimado que el SAOS aumenta el riesgo de IC en OR 2.38 (IC 95\% 1.22-4.64). ${ }^{1}$

Por otro lado, en varios estudios el uso de CPAP han mostrado tener beneficio en pacientes con SAOS e IC. Los resultados de un metaanálisis indican que el tratamiento produce un aumento de 3.5\% en la fracción de eyección del ventrículo izquierdo (FEVI) de los pacientes con SAOS e IC después de recibir terapia con CPAP. ${ }^{60}$

\section{Trastornos del ritmo}

La hipoxia intermitente puede causar alteraciones de la frecuencia cardíaca: aumento o disminución según el predominio de actividad parasimpática o simpática ${ }^{61}$ Algunos estudios han mostrado que las alteraciones en el ritmo cardíaco son una complicación importante en el SAOS, uno de ellos es el realizado por Simantirakis et al. quienes observaron alteraciones del ritmo cardíaco en la mitad de los pacientes con SAOS. ${ }^{62}$

Otro punto importante es la correlación existente entre la alteración del ritmo cardíaco con la desaturación y gravedad de la apnea como fue demostrado en el INCMNSZ, donde se identificó que el riesgo para desarrollar arritmias cardíacas incrementa con la severidad del SAOS y con el grado de desaturación. ${ }^{26}$

Por otro lado, Tanigawa et al. demostraron que la severidad de la hipoxemia se asocia con fibrilación auricular (FA).$^{63}$ El estudio de Sleep Heart Health Study enfatizó que la FA es significativamente más frecuente en los individuos con SAOS contra los que no lo tienen, $4.8 \%$ vs. $0.9 \%$, respectivamente. ${ }^{64}$ Gami et al. estimaron OR 2.57 del riesgo aumentado de muerte súbita en los pacientes con SAOS. ${ }^{65} \mathrm{La}$ terapia con CPAP ha mostrado utilidad en pacientes posablación donde el riesgo disminuye de manera significante. ${ }^{66}$

\section{ASOCIACIÓN DEL SAOS CON HIPERTENSIÓN PULMONAR}

\section{Definición y clasificación de hipertensión pulmonar}

La HP es una condición cardiopulmonar de diversas etiologías y mecanismos fisiopatológicos que conllevan a la disfunción ventricular derecha ${ }^{67}$ La presión media de la arteria pulmonar (mPAP), medida por cateterismo cardíaco derecho se considera normal de 8 a $20 \mathrm{mmHg}$, por arriba de $25 \mathrm{mmHg}$ en reposo se considera HP. 68

De acuerdo con las características etiológicas y terapéuticas la HP se clasifica en los siguientes grupos: 1) hipertensión arterial pulmonar, 2) HP secundaria a enfermedad cardiovascular izquierda, 3) HP secundaria a enfermedad pulmonar y/o hipoxemia, 4) HP debida a enfermedad tromboembólica pulmonar y 5) HP de origen indeterminado o multifactorial. ${ }^{69}$

EI SAOS pertenece al tercer grupo debido a que la hipoxemia crónica, secundaria a los períodos de obstrucción de la vía aérea durante el sueño, se considera la etiología de HP en estos pacientes. El aumento de presión en la arteria pulmonar genera resistencia al flujo y produce mayor carga para el VD. Esta condición de forma crónica produce en el ventrículo derecho modificaciones estructurales (figura 3) y funcionales que deterioran el pronóstico de estos pacientes. 


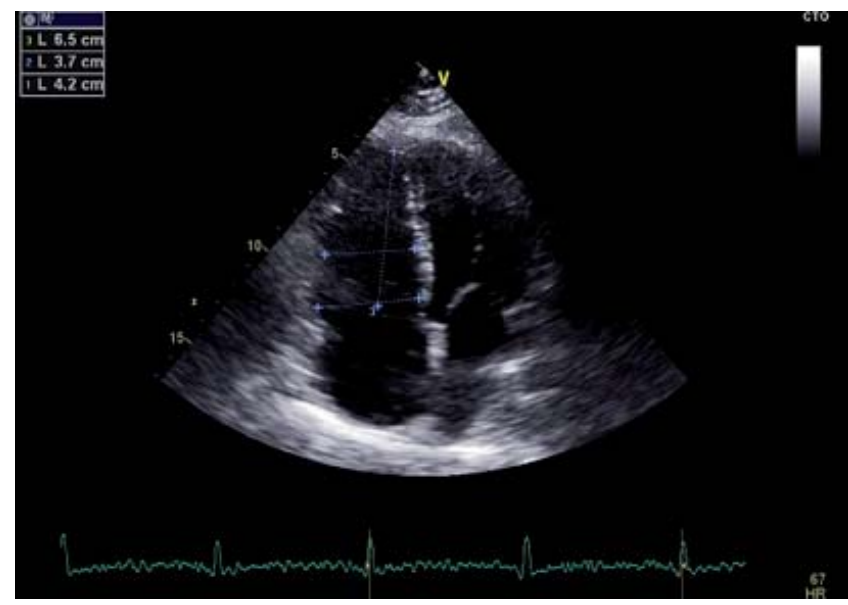

Figura 3. Ecocardiograma transtorácico en corte de cuatro cámaras. Se observan datos de repercusión hemodinámica secundarios a hipertensión pulmonar que incluyen aumento de tamaño y dilatación del ventrículo derecho $(3.7 \mathrm{~cm}$ de diámetro medio).

\section{Prevalencia de HP en SAOS}

Estudios epidemiológicos muestran que la prevalencia de HP en pacientes con SAOS es del 17 al 53\%. Chaouat et al. en 1996 mediante cateterismo cardíaco evaluaron a 220 pacientes con SAOS y encontraron HP en 37 de ellos, una prevalencia del $17 \% .{ }^{70} \mathrm{~A}$ su vez, el equipo de Nijjima evaluó 19 pacientes con SAOS y encontró HP en 10, una prevalencia de 53\%. ${ }^{71}$

En un estudio reciente de corte transversal en pacientes con HP en los que se estudiaron trastornos respiratorios durante el sueño (TRS) como apnea obstructiva del sueño, apnea central e hipoventilación relacionado al sueño, se documentó una prevalencia del $71 \%$ de TRS, presentándose SAOS en el $56 \%$ de los pacientes. ${ }^{72}$ Estos datos son relevantes debido a que los pacientes con SAOS e HP tienen una tasa de mortalidad más alta que los pacientes con SAOS sin HP. ${ }^{73}$ El tratamiento de los TRS es de importancia en el paciente con HP y SAOS.

\section{Impacto de SAOS en HP}

La hipoxia es un factor común en los trastornos respiratorios crónicos. Mediante experimentos de hipoxia intermitente en modelos animales se ha demostrado que este estado produce aumento de la presión arterial pulmonar. En roedores sometidos a hipoxia cada 30 segundos durante ocho horas al día por cinco días de la semana durante cinco semanas, se encontró aumento significativo de la presión arterial pulmonar. Además, aumento de tamaño del VD y del hematocrito. ${ }^{74} \mathrm{Sin}$ embargo, en humanos parece difícil identificar el verdadero impacto de SAOS como causa independiente de HP debido a la existencia de comorbilidades existentes como obesidad y enfermedad pulmonar obstructiva crónica.

La respuesta a esta pregunta parece acercarse mediante estudios que evalúan el impacto terapéutico sobre la HP en pacientes con SAOS. El estudio de Alchanatis et al. estimó la presión sistólica de la arteria pulmonar (sPAP) mediante ecografía en pacientes con SAOS antes y después de seis semanas del tratamiento con CPAP y encontraron en sus resultados un descenso significativo de la SPAP. ${ }^{14}$ Sajkov et al. mostraron mediante ecografía la disminución significativa de la sPAP en pacientes con HP leve en cuatro semanas de tratamiento con CPAP. ${ }^{75}$ Otro estudio de Motta et al. describe a pacientes con SAOS antes y después de realizarles traqueostomía terapéutica, en los resultados se observó un descenso significativo de PAP $50 \% .^{76}$ En este punto es conveniente mencionar también que existen estudios como el realizado por Sforza et al. en el que se observó disminución de la PAP no significativa al evaluar pacientes con SAOS e HP después de recibir tratamiento con CPAP por un año. ${ }^{77}$

Por otra parte, un estudio reciente de metaanálisis mostró que el tratamiento con CPAP induce una reducción estadísticamente significativa en la presión arterial pulmonar en pacientes con SAOS (diferencia promedio estandarizada de $-1.34, p=0.009$ ). Aunque dicho metaanálisis cuenta con limitaciones se muestra que el tratamiento con CPAP se asocia con una disminución en la SPAP en pacientes con SAOS. ${ }^{78}$

\section{CONCLUSIÓN}

La importancia del diagnóstico y tratamiento del SAOS ha sido evidenciada en las recientes publicaciones que lo muestran como factor de riesgo independiente para el desarrollo de enfermedades cardiovasculares.

En nuestro medio la prevalencia del SAOS está subestimada debido a que la mayoría de los pacientes que acuden a las clínicas del sueño representan la forma más evidente de la enfermedad, por lo que es necesario realizar estudios poblacionales en los que se pueda reflejar el impacto del SAOS en la población general.

Se reconoce que la hipoxia es el principal factor detonante de disfunción endotelial y como consecuencia facilita la aterogénesis y el desarrollo de comorbilidades que se presentan como las principales causas de muerte en nuestro país. Es importante documentar el desarrollo de HP en estos pacientes, pues se sabe que sin tratamiento evolucionan a disfunción ventricular, la cual continúa teniendo alta mortalidad a pesar de las medidas terapéuticas actuales. 
El tratamiento mediante presión positiva resulta efectivo en la mayoría de los informes publicados, aunque se tiene el dato de que no existe un beneficio significativo en prevención secundaria de eventos cardiovasculares. No obstante, se debe hacer un esfuerzo encaminado a favorecer la prevención.

Por último, es importante mencionar que los estudios diseñados para demostrar el impacto del SAOS retan a los investigadores a seguir desarrollando estrategias que permitan conocer con mayor precisión y certidumbre la asociación entre enfermedad cardíaca y alteraciones respiratorias durante el sueño. No debemos olvidar que en la práctica clínica los pacientes se presentan con comorbilidades que nos obligan a buscar la mejor estrategia para un tratamiento integral.

\section{Agradecimientos}

Al personal del Departamento de Ecocardiografía y de la Clínica de Trastornos del Dormir del INCMNSZ.

\section{REFERENCIAS}

1. Shahar E, Whitney CW, Redline S, et al. Sleep-disordered breathing and cardiovascular disease: cross-sectional results of the Sleep Heart Health Study. Am J Respir Crit Care Med 2001;163(1):19-25.

2. Somers VK, White DP, Amin R, et al.; American Heart Association Council for High Blood Pressure Research Professional Education Committee, Council on Clinical Cardiology; American Heart Association Stroke Council; American Heart Association Council on Cardiovascular Nursing; American College of Cardiology Foundation. Sleep apnea and cardiovascular disease: an American Heart Association/American College Of Cardiology Foundation Scientific Statement from the American Heart Association Council for High Blood Pressure Research Professional Education Committee, Council on Clinical Cardiology, Stroke Council, and Council On Cardiovascular Nursing. In collaboration with the National Heart, Lung, and Blood Institute National Center on Sleep Disorders Research (National Institutes of Health). Circulation 2008;118(10):10801111. doi: 10.1161/CIRCULATIONAHA.107.189375.

3. Floras JS. Sleep apnea and cardiovascular risk. J Cardiol 2014;63(1):3-8. doi: 10.1016/j.jjcc.2013.08.009.

4. Peppard PE, Young T, Palta M, Skatrud J. Prospective study of the association between sleep-disordered breathing and hypertension. N Engl J Med 2000;342(19):1378-1384.

5. Li M, Hou WS, Zhang XW, Tang ZY. Obstructive sleep apnea and risk of stroke: a meta-analysis of prospective studies. Int J Cardiol 2014;172(2):466-469. doi: 10.1016/j. ijcard.2013.12.230.
6. Hohl M, Linz B, Böhm M, Linz D. Obstructive sleep apnea and atrial arrhythmogenesis. Curr Cardiol Rev 2014;10(4):362-368.

7. Kasai T. Sleep apnea and heart failure. J Cardiol 2012;60(2):78-85. doi: 10.1016/j.jjcc.2012.05.013.

8. Sajkov D, McEvoy RD. Obstructive sleep apnea and pulmonary hypertension. Prog Cardiovasc Dis 2009;51(5):363-370. doi: 10.1016/j.pcad.2008.06.001.

9. Loke YK, Brown JW, Kwok CS, Niruban A, Myint PK. Association of obstructive sleep apnea with risk of serious cardiovascular events: a systematic review and meta-analysis. Circ Cardiovasc Qual Outcomes 2012;5(5):720-728.

10. Gonzaga C, Bertolami A, Bertolami M, Amodeo C, Calhoun D. Obstructive sleep apnea, hypertension and cardiovascular diseases. J Hum Hypertens 2015;29(12):705-712. doi: 10.1038/jhh.2015.15

11. Lattimore JD, Celermajer DS, Wilcox I. Obstructive sleep apnea and cardiovascular disease. J Am Coll Cardiol 2003;41(9):1429-1437.

12. Korcarz CE, Peppard PE, Young TB, et al. Effects of obstructive sleep apnea and obesity on cardiac remodeling: The Wisconsin Sleep Cohort Study. Sleep 2016;39(6):1187-1195. doi: 10.5665/sleep.5828.

13. Dong JY, Zhang YH, Qin LQ. Obstructive sleep apnea and cardiovascular risk: meta-analysis of prospective cohort studies. Atherosclerosis 2013;229(2):489-495. doi: 10.1016/j.atherosclerosis.2013.04.026.

14. Alchanatis M, Tourkohoriti G, Kakouros S, Kosmas E, Podaras S, Jordanoglou JB. Daytime pulmonary hypertension in patients with obstructive sleep apnea: the effect of continuous positive airway pressure on pulmonary hemodynamics. Respiration 2001;68(6):566-572.

15. Dewan NA, Nieto FJ, Somers VK. Intermittent hypoxemia and OSA: implications for comorbidities. Chest 2015;147(1):266-274. doi: 10.1378/chest.14-0500.

16. American Academy of Sleep Medicine. International classification of sleep disorders. 3rd ed. Darien II: American Academy of Sleep Medicine; 2014.

17. Young T. Rationale, design and findings from the Wisconsin Sleep Cohort Study: Toward understanding the total societal burden of sleep disordered breathing. Sleep Med Clin 2009;4(1):37-46.

18. Hansel B, Cohen-Aubart F, Dourmap C, Giral P, Bruckert $\mathrm{E}$, Girerd X. [Prevalence of sleep apnea in men with metabolic syndrome and controlled hypertension]. Arch Mal Coeur Vaiss 2007;100(8):637-641.

19. Drager LF, Genta PR, Pedrosa RP, et al. Characteristics and predictors of obstructive sleep apnea in patients with systemic hypertension. Am J Cardiol 2010;105(8):11351139. doi: 10.1016/j.amjcard.2009.12.017.

20. Logan AG, Perlikowski SM, Mente A, et al. High prevalence of unrecognized sleep apnoea in drugresistant hypertension. J Hypertens 2001;19(12):22712277.

21. Gami AS, Pressman G, Caples SM, et al. Association of atrial fibrillation and obstructive sleep apnea. Circulation 2004;110(4):364-367. 
22. Drager LF, Lopes HF, Maki-Nunes C, et al. The impact of obstructive sleep apnea on metabolic and inflammatory markers in consecutive patients with metabolic syndrome. PLoS One 2010;5(8):e12065. doi: 10.1371/ journal.pone.0012065.

23. Punjabi NM. The epidemiology of adult obstructive sleep apnea. Proc Am Thorac Soc 2008;5(2):136-143. doi: 10.1513/pats.200709-155MG.

24. Schwartz AR, Patil SP, Laffan AM, Polotsky V, Schneider $\mathrm{H}$, Smith PL. Obesity and obstructive sleep apnea: pathogenic mechanisms and therapeutic approaches. Proc Am Thorac Soc 2008;5(2):185-192. doi: 10.1513/ pats.200708-137MG.

25. Bouscoulet LT, Vázquez-García JC, Muino A, et al.; PLATINO Group. Prevalence of sleep related symptoms in four Latin American cities. J Clin Sleep Med 2008;4(6):579-585.

26. Valencia-Flores M, Orea A, Castano VA, et al. Prevalence of sleep apnea and electrocardiographic disturbances in morbidly obese patients. Obes Res 2000;8(3):262269.

27. Bloch KE, Latshang TD, Ulrich S. Patients with obstructive sleep apnea at altitude. High Alt Med Biol 2015;16(2):110-116. doi: 10.1089/ham.2015.0016.

28. Valencia-Flores M, Mokhlesi B, Santiago-Ayala V, et al. Intermittent hypoxemia and sleep fragmentation: associations with daytime alertness in obese sleep apnea patients living at moderate altitude. Sleep Med 2016;20:103-109. doi: 10.1016/j.sleep.2016.01.002

29. Bazurto Zapata MA, Martínez-Guzmán W, VargasRamírez L, Herrera K, González-García M. Prevalence of central sleep apnea during continuous positive airway pressure (CPAP) titration in subjects with obstructive sleep apnea syndrome at an altitude of 2640 m. Sleep Med 2015;16(3):343-346. doi: 10.1016/j. sleep.2014.09.022

30. Latshang TD, Nussbaumer-Ochsner Y, Henn RM, et al. Effect of acetazolamide and autoCPAP therapy on breathing disturbances among patients with obstructive sleep apnea syndrome who travel to altitude: a randomized controlled trial. JAMA 2012;308(22):23902398. doi: 10.1001/jama.2012.94847.

31. Wang X, Ouyang Y, Wang Z, Zhao G, Liu L, Bi Y. Obstructive sleep apnea and risk of cardiovascular disease and all-cause mortality: a meta-analysis of prospective cohort studies. Int J Cardiol 2013;169(3):207214. doi: 10.1016/j.ijcard.2013.08.088.

32. Peker $Y$, Kraiczi H, Hedner J, Loth $S$, Johansson A, Bende M. An independent association between obstructive sleep apnoea and coronary artery disease. Eur Respir J 1999;14(1):179-184.

33. Badran M, Ayas N, Laher I. Cardiovascular complications of sleep apnea: role of oxidative stress. Oxid Med Cell Longev 2014;2014:985258. doi: 10.1155/2014/985258.

34. Israel LP, Benharoch D, Gopas J, Goldbart AD. A pro-inflammatory role for nuclear factor kappa $B$ in childhood obstructive sleep apnea syndrome. Sleep 2013;36(12):1947-1955. doi: 10.5665/sleep.3236.

35. Ozkan Y, Firat H, Simşek B, Torun M, YardimAkaydin S. Circulating nitric oxide (NO), asymmetric dimethylarginine (ADMA), homocysteine, and oxidative status in obstructive sleep apnea-hypopnea syndrome (OSAHS). Sleep Breath 2008;12(2):149-154.

36. Ciccone MM, Scicchitano P, Zito A, et al. Correlation between inflammatory markers of atherosclerosis and carotid intima-media thickness in obstructive sleep apnea. Molecules 2014;19(2):1651-1662. doi: 10.3390/ molecules19021651.

37. Cho ER, Kim H, Seo HS, Suh S, Lee SK, Shin C. Obstructive sleep apnea as a risk factor for silent cerebral infarction. J Sleep Res 2013;22(4):452-458. doi: 10.1111/jsr.12034.

38. Yokoe T, Minoguchi K, Matsuo H, et al. Elevated levels of C-reactive protein and interleukin- 6 in patients with obstructive sleep apnea syndrome are decreased by nasal continuous positive airway pressure. Circulation 2003;107(8):1129-1134.

39. Drager LF, Bortolotto LA, Figueiredo AC, Krieger EM, Lorenzi GF. Effects of continuous positive airway pressure on early signs of atherosclerosis in obstructive sleep apnea. Am J Respir Crit Care Med 2007;176(7):706712.

40. McEvoy RD, Antic NA, Heeley E, et al.; SAVE Investigators and Coordinators. CPAP for prevention of cardiovascular events in obstructive sleep apnea. N Engl J Med 2016;375(10):919-931. doi: 10.1056/ NEJMoa1606599.

41. Lim S, Barter P. Antioxidant effects of statins in the management of cardiometabolic disorders. J Atheroscler Thromb 2014;21(10):997-1010.

42. Gonzaga C, Bertolami A, Bertolami M, Amodeo C, Calhoun D. Obstructive sleep apnea, hypertension and cardiovascular diseases. J Hum Hypertens 2015;29(12):705-712. doi: 10.1038/jhh.2015.15.

43. Pedrosa RP, Drager LF, Gonzaga CC, et al. Obstructive sleep apnea: the most common secondary cause of hypertension associated with resistant hypertension. Hypertension 2011;58(5):811-817. doi: 10.1161/ HYPERTENSIONAHA.111.179788.

44. Nieto FJ, Young TB, Lind BK, et al. Association of sleepdisordered breathing, sleep apnea, and hypertension in a large community-based study. Sleep Heart Health Study. JAMA 2000;283(14):1829-1836.

45. Baguet JP, Barone-Rochette G, Pépin JL. Hypertension and obstructive sleep apnoea syndrome: current perspectives. J Hum Hypertens 2009;23(7):431-443. doi: 10.1038/jhh.2008.147

46. Gonzaga CC, Gaddam KK, Ahmed MI, et al. Severity of obstructive sleep apnea is related to aldosterone status in subjects with resistant hypertension. J Clin Sleep Med 2010;6(4):363-368.

47. Becker HF, Jerrentrup A, Ploch $\mathrm{T}$, et al. Effect of nasal continuous positive airway pressure treatment on blood pressure in patients with obstructive sleep apnea. Circulation 2003;107(1):68-73.

48. Fava C, Dorigoni S, Dalle Vedove F, et al. Effect of CPAP on blood pressure in patients with OSA/ hypopnea a systematic review and meta-analysis. Chest 2014;145(4):762-771. 
49. Mohsenin V. Obstructive sleep apnea: a new preventive and therapeutic target for stroke: a new kid on the block. Am J Med 2015;128(8):811-816. doi: 10.1016/j. amjmed.2015.01.037

50. Yaggi HK, Concato J, Kernan WN, Lichtman JH, Brass LM, Mohsenin V. Obstructive sleep apnea as a risk factor for stroke and death. N Engl J Med 2005;353(19):2034-2041.

51. Arzt M, Young T, Finn L, Skatrud JB, Bradley TD. Association of sleep-disordered breathing and the occurrence of stroke. Am J Respir Crit Care Med 2005;172(11):1447-1451.

52. Parra O, Sánchez-Armengol A, Capote F, et al. Efficacy of continuous positive airway pressure treatment on 5-year survival in patients with ischaemic stroke and obstructive sleep apnea: a randomized controlled trial. J Sleep Res 2015;24(1):47-53. doi: 10.1111/jsr.12181.

53. Martínez-García MA, Soler-Cataluna JJ, EjarqueMartínez L, et al. Continuous positive airway pressure treatment reduces mortality in patients with ischemic stroke and obstructive sleep apnea: a 5-year follow-up study. Am J Respir Crit Care Med 2009;180(1):36-41. doi: 10.1164/rccm.200808-13410C.

54. Shahar E, Lee S, Kim J, Duval S, Barber C, Luepker RV. Hospitalized heart failure: rates and long-term mortality. J Card Fail 2004;10(5):374-379.

55. Costanzo MR, Khayat R, Ponikowski P, et al. Mechanisms and clinical consequences of untreated central sleep apnea in heart failure. J Am Coll Cardiol 2015;65(1):7284. doi: 10.1016/j.jacc.2014.10.025.

56. Bradley TD, Hall MJ, Ando S, Floras JS. Hemodynamic effects of simulated obstructive apneas in humans with and without heart failure. Chest 2001;119(6):18271835.

57. Stoohs R, Guilleminault C. Cardiovascular changes associated with obstructive sleep apnea syndrome. J Appl Physiol (1985). 1992;72(2):583-589.

58. Brinker JA, Weiss JL, Lappe DL, et al. Leftward septal displacement during right ventricular loading in man. Circulation 1980;61(3):626-633.

59. Tolle FA, Judy WV, Yu PL, Markand ON. Reduced stroke volume related to pleural pressure in obstructive sleep apnea. J Appl Physiol Respir Environ Exerc Physiol 1983;55(6):1718-1724.

60. Sun H, Shi J, Li M, Chen X. Impact of continuous positive airway pressure treatment on left ventricular ejection fraction in patients with obstructive sleep apnea: a meta-analysis of randomized controlled trials. PLoS One 2013;8(5):e62298. doi: 10.1371/journal. pone.0062298.

61. Leung RS. Sleep-disordered breathing: autonomic mechanisms and arrhythmias. Prog Cardiovasc Dis 2009;51(4):324-338. doi: 10.1016/j. pcad.2008.06.002.

62. Simantirakis EN, Schiza SI, Marketou ME, et al. Severe bradyarrhythmias in patients with sleep apnoea: the effect of continuous positive airway pressure treatment: a long-term evaluation using an insertable loop recorder. Eur Heart J 2004;25(12):1070-1076.
63. Tanigawa T, Yamagishi K, Sakurai S, et al. Arterial oxygen desaturation during sleep and atrial fibrillation. Heart 2006;92(12):1854-1855.

64. Mehra R, Benjamin EJ, Shahar E, et al.; Sleep Heart Health Study. Association of nocturnal arrhythmias with sleep-disordered breathing: The Sleep Heart Health Study. Am J Respir Crit Care Med 2006;173(8):910-916.

65. Gami AS, Howard DE, Olson EJ, Somers VK. Day-night pattern of sudden death in obstructive sleep apnea. N Engl J Med 2005;352(12):1206-1214.

66. Fein AS, Shvilkin A, Shah D, et al. Treatment of obstructive sleep apnea reduces the risk of atrial fibrillation recurrence after catheter ablation. J Am Coll Cardiol 2013;62(4):300-305. doi: 10.1016/j. jacc.2013.03.052.

67. Aronson D, Darawsha W, Atamna A, et al. Pulmonary hypertension, right ventricular function, and clinical outcome in acute decompensated heart failure. J Card Fail 2013;19(10):665-671. doi: 10.1016/j. cardfail.2013.08.007.

68. Hoeper MM, Bogaard HJ, Condliffe R, et al. Definitions and diagnosis of pulmonary hypertension. J Am Coll Cardiol 2013;62(25 Suppl):D42-D50. doi: 10.1016/j. jacc.2013.10.032.

69. Simonneau G, Gatzoulis MA, Adatia I, et al. [Updated clinical classification of pulmonary hypertension]. Turk Kardiyol Dern Ars 2014;42 Suppl 1:45-54.

70. Chaouat A, Weitzenblum E, Krieger J, Oswald M, Kessler R. Pulmonary hemodynamics in the obstructive sleep apnea syndrome. Results in 220 consecutive patients. Chest 1996;109(2):380-386.

71. Niijima $\mathrm{M}$, Kimura $\mathrm{H}$, Edo $\mathrm{H}$, et al. Manifestation of pulmonary hypertension during REM sleep in obstructive sleep apnea syndrome. Am J Respir Crit Care Med 1999;159(6):1766-1772.

72. Minic M, Granton JT, Ryan CM. Sleep disordered breathing in group 1 pulmonary arterial hypertension. J Clin Sleep Med 2014;10(3):277-283. doi: 10.5664/ jcsm. 3528 .

73. Minai OA, Ricaurte B, Kaw R, et al. Frequency and impact of pulmonary hypertension in patients with obstructive sleep apnea syndrome. Am J Cardiol 2009;104(9):13001306. doi: 10.1016/j.amjcard.2009.06.048.

74. McGuire M, Bradford A. Chronic intermittent hypercapnic hypoxia increases pulmonary arterial pressure and haematocrit in rats. Eur Respir J 2001;18(2):279-285.

75. Sajkov D, Wang T, Saunders NA, Bune AJ, McEvoy $\mathrm{RD}$. Continuous positive airway pressure treatment improves pulmonary hemodynamics in patients with obstructive sleep apnea. Am J Respir Crit Care Med 2002;165(2):152-158.

76. Motta J, Guilleminault C, Schroeder JS, Dement WC. Tracheostomy and hemodynamic changes in sleepinducing apnea. Ann Intern Med 1978;89(4):454458.

77. Sforza E, Krieger J, Weitzenblum E, Apprill M, Lampert E, Ratamaharo J. Long-term effects of treatment with nasal continuous positive airway pressure on daytime lung 
function and pulmonary hemodynamics in patients with obstructive sleep apnea. Am Rev Respir Dis 1990;141(4 Pt 1):866-870.

78. Sun X, Luo J, Xiao Y. Continuous positive airway pressure is associated with a decrease in pulmonary artery pressure in patients with obstructive sleep apnoea: a meta-analysis. Respirology 2014;19(5):670-674. doi: 10.1111/resp.12314. $\triangle$ Correspondencia:

Dr. Jaime Eduardo Morales-Blanhir

Instituto Nacional de Ciencias Médicas y Nutrición Salvador Zubirán.

Vasco de Quiroga Núm. 15, Colonia Sección XVI, 14080, Tlalpan, Ciudad de México.

Correo electrónico: moralesjaime@usa.net

Los autores declaran no tener conflicto de intereses. 